\title{
Soret effect of mono- di- and tri-glycols in ethanol
}

\section{Michael Klein* and Simone Wiegand}

\author{
Received Xth XXXXXXXXXX 20XX, Accepted Xth $X X X X X X X X X 20 X X$ \\ First published on the web $X$ th $X X X X X X X X X X 200 X$ \\ DOI: 10.1039/b000000x
}

We employed thermal diffusion forced Rayleigh scattering (TDFRS) to investigate the chain length dependence of the thermal diffusion behavior of short glycols in ethanol. We studied three glycols, monoethylenglycol (MEG), diethylenglycol (DEG) and triethylenglycol (TEG) in an ethanol solution. In contrast to the polymer polyethylenglycol, the shorter glycols used within the framework of this study are completely soluble in ethanol, at least for low molar fractions. In order to guarantee a good solubility of the glycols, the measurements were limited to molar fractions of $10 \%$ and $20 \%$. As the different glycols only differ by their chain length it will be possible to deduce its influence on the thermal diffusion properties. Due to the fairly short persistence length of the glycols, $D_{T}$ reaches already for the dimer a plateau value. This finding agrees with simulation results. Furthermore, the thermal expansion and the kinematic viscosity are measured to investigate if empirical correlations seen for other systems could be confirmed.

\section{Introduction}

Although there is large activity within the research community, the Ludwig-Soret effect, a non-equilibrium process taking place when initially at least binary mixtures are exposed to a temperature gradient, is still not understood. Due to a temperature gradient a mass flow, leading to a concentration gradient is initiated. For binary mixtures this can be described by the Soret-coefficient $S_{\mathrm{T}}=D_{\mathrm{T}} / D$, which is defined as the ratio between the thermal diffusion coefficient $D_{\mathrm{T}}$ and the mass diffusion coefficient $D$. From the applications side of view the effect is interesting for example in the fields of biology ${ }^{1,2}$, energy ${ }^{3}$ and oil industry ${ }^{4}$.

For many binary liquid mixtures, many experimental, theoretical and simulation attempts to explain the mechanism of thermodiffusion have been made ${ }^{5-14}$. A relation of the governing coefficients with physical properties such as density ${ }^{13}$, molecular weight ${ }^{14}$, moment of inertia ${ }^{5}$, viscosity ${ }^{15}$, thermal expansion ${ }^{15}$, molecular structure ${ }^{11}$, and also chemical interactions ${ }^{12}$ have been proposed. Apart from low molecular weight mixtures also polymers solutions and colloidal suspensions in polar and non-polar solvents have been investigated $^{16-18}$. For this study, the thermal expansion coefficient $\alpha$ and the kinematic viscosity $v$ are measured to compare to recent results on alkanes ${ }^{15}$ and sugars ${ }^{19,20}$.

A few experimental, theoretical and simulation studies have been performed to investigate the chain length dependence of $D_{\mathrm{T}}$. Based on an early work by Brochard and de Gennes ${ }^{21}$, Würger ${ }^{22}$ showed that the solvent molecular structure and the solute Brownian motion give rise to a contribution to the overall thermophoretic velocity. He states that this value is al-

\footnotetext{
${ }^{a}$ Forschungszentrum Juelich GmbH, IFF - Soft Matter, D-52428 Juelich
}

ways negative. This model leads to an increase of $D_{\mathrm{T}}$ with increasing chain length and a plateau value for higher chain lengths. This approach describes n-alkanes and polystyrene as nicely shown by Stadelmaier et al. ${ }^{23}$. Nevertheless a study by Blanco et al. on oligosaccharides ${ }^{20}$ shows exactly the opposite trend: The thermal diffusion $D_{T}$ decreases with increasing chain length until it saturates. The saturation behavior has also been found in simulations. Zhang and Müller-Plathe ${ }^{24}$ showed by reverse nonequilibrium molecular dynamics simulations, employing a simple bead-spring model that the chain length for which the plateau value is reached, depends on the flexibility of the chains. In the simulations, more flexible chains reach a constant $D_{\mathrm{T}}$ at shorter chain lengths compared to rigid chains. Describing the chain length in terms of the persistence length $l_{p}, D_{\mathrm{T}}$ becomes constant when the chain length is of around two to three times $l_{p}$. The chain length dependence of $D_{\mathrm{T}}$ of alkanes ${ }^{14}$, polystyrene ${ }^{23}$, sugars ${ }^{20}$ and also of glycols in aqueous solution ${ }^{25}$ is already documented in literature. If we follow the argumentation of the simulation results we expect for glycols in ethanol a different behavior compared the aqueous solutions, because in contrast to water no hydration layer is formed. This should lead to a higher flexibility of the molecules and a shorter persistence length and therefore $D_{\mathrm{T}}$ should reach its plateau value already for shorter chains.

An additional motivation to study the chain length dependence of glycols came from a study of polyethylenglycol $(\mathrm{PEG})$ in ethanol water mixtures ${ }^{26}$. As illustrated in Fig. 1 the system shows an interesting behavior at a water weight fraction between $w=5-70 \%$. In this range PEG and ethanol enrich at the warm side, while between $w=70-82 \%$ PEG goes to the warm side and ethanol to the cold. For higher water contents both go to the cold side ${ }^{26}$. This makes it extremely 


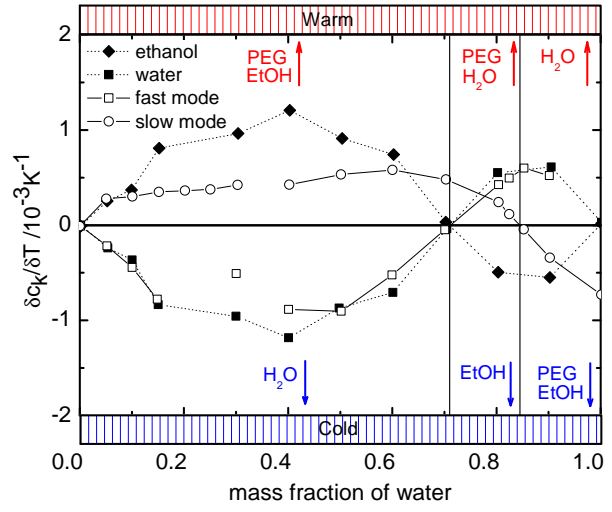

Fig. 1 Mass fraction differentials $\delta c / \delta T$ of the binary mixtures water/ ethanol, $c w, 1-w$, and the ternary mixtures $\mathrm{PEO} /$ water/ethanol, $\mathrm{c} c c, w, 1-c-w$, as a function of solvent composition. Closed and open symbols represent data for the binary and ternary systems, respectively. The lines are guides to the eye. Data from ${ }^{26}$.

difficult to perform convection free experiments. In the case of a binary mixture it is usually possible to find a situation that the denser component moves to the bottom, but in the case of a ternary mixture with the two sign change concentration it will be impossible. This behavior makes this mixture in general extremely appealing as a benchmark system for microgravitational experiments. One drawback is the slow diffusing polymer, which might decompose upon longtime storage during space experiments and which also requires very long measurement times, which are often not available. Therefore it is interesting to investigate shorter glycols, which have a better longtime stability and require shorter measurement times.

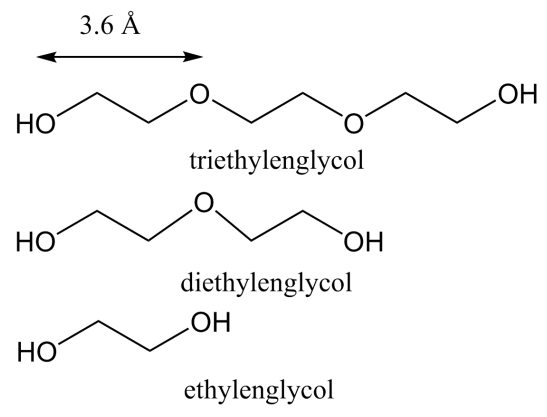

Fig. 2 Structure of the investigated glycols.

To investigate the chain length dependence of the glycols, solutions of mono-, di- and triethylenglycol (see Fig. 2 in ethanol were measured. To improve the signal to noise ratio the regime of measurements was limited to molar concentra- tions of $10 \%$ and $20 \%$ and a temperature regime from $30{ }^{\circ} \mathrm{C}$ to $50{ }^{\circ} \mathrm{C}$.

\section{Experimental section}

\subsection{Thermal Diffusion Forced Rayleigh Scattering}

The main experimental technique employed in this study was Thermal Diffusion Forced Rayleigh Scattering (TDFRS). This technique is intensively described elsewhere ${ }^{27-29}$. The setup uses a blue laser $\left(\lambda_{w}=488 \mathrm{~nm}\right)$, which is splitted into two writing beams of equal intensity, which are aligned in such a way that they interfere within the sample cell. Thus, they form an intensity grating, which is due to a small amount of dye Quinizarin $^{\circledR}$ in the sample solution, converted into a temperature grating. This is in turn also causing a concentration grating by the effect of thermal diffusion. Both gratings add up to a refractive index grating which is detected by a third laser beam $\left(\lambda_{r}=633 \mathrm{~nm}\right)$. For the determination of the transport coefficients, the measured heterodyne signal is fitted using the independently measured contrast factors $(\partial n / \partial c)_{p, T}$ and $(\partial n / \partial T)_{p, c}$.

\subsection{Materials and Equipment}

Ethylenglycol $(\geq 99.8 \%)$, diethylenglycol $(\geq 99 \%)$, triethylenglycol $(\geq 99 \%)$ were purchased at Sigma Aldrich. Ethanol of $99.9 \%$ purity by Merck was used. The mass concentrations were prepared employing a Mettler-Toledo digital scale with an accuracy of $0.0001 \mathrm{~g}$. To convert the light energy into heat energy, Quinizarin ${ }^{\circledR}$ was added to the solution. An optical quartz cell by Hellma with an optical path length of about $0.2 \mathrm{~mm}$ was filled through a filter (Spartam 13/0.45 RC). An Anton Paar automatic Microviscometer AMVn was used to determine the kinematic viscosity of the mixtures. To determine the density $\rho$ and the thermal expansion coefficient $\alpha$ an Anton Paar DMA 4500 densimeter was used. It has an accuracy of $\Delta \rho= \pm 0.00001 \mathrm{~g} \mathrm{~cm}^{-3}$ and a temperature control of $\Delta T= \pm 0.01 \mathrm{~K}$. Employing an Anton Paar RXA 156 refractometer the refractive index as function of mass concentration has been determined. The accuracy is of $\Delta n= \pm 0.00003$ with a temperature control of $\Delta T= \pm 0.01 \mathrm{~K}$. For each solution five different mass concentrations have been prepared and measured. By linear interpolation the increment in refractive index $\partial n / \partial c$ was determined. The refractive index increment with temperature $(\partial n / \partial T)$ has been measured employing a Michelson interferometer in a temperature range $2 \mathrm{~K}$ below and above the desired temperature.

\subsection{Experiments and Discussion}

The glycols studied in this work have been prepared as $10 \%$ and $20 \%$ molar solutions. The temperature has been varied 


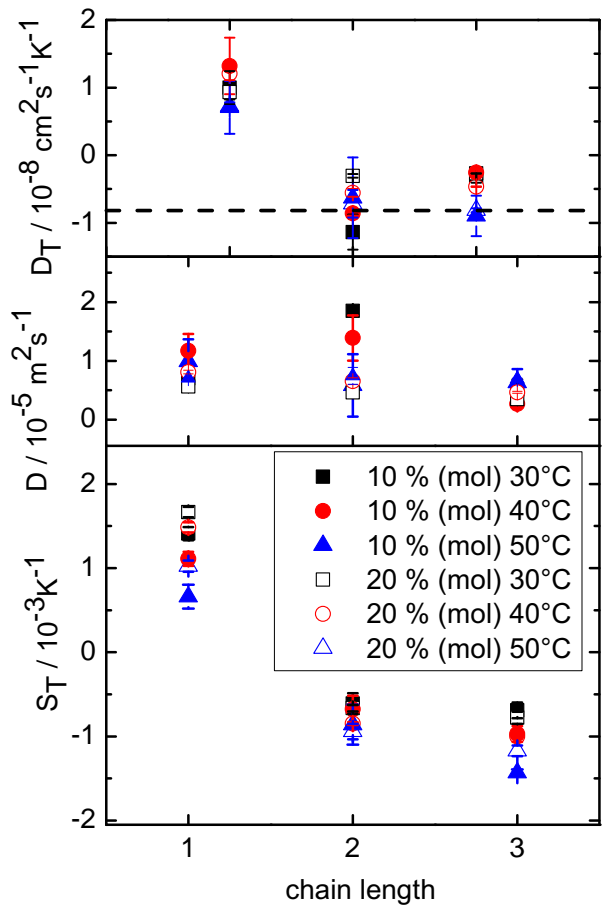

Fig. 3 Thermal diffusion coefficient $D_{T}$, mass diffusion $D$ and Soret coefficient $S_{\mathrm{T}}$ plotted as function of chain length. It can be seen that the sign of $D_{T}$ and $S_{\mathrm{T}}$ changes between the monomer and the dimer. The different molar concentrations of $10 \%$ (closed symbols) and $20 \%$ (open symbols) provide results which are equal within error. The dashed line in the $D_{T}$ plot denotes the value of polyethyleneglycol in solution with ethanol and water as reported in 26 .

between $30{ }^{\circ} \mathrm{C}$ and $50{ }^{\circ} \mathrm{C}$. In Fig. 3 the chain length dependence of the thermal diffusion coefficient $D_{T}$, the mass diffusion coefficient $D$ and the Soret coefficient $S_{\mathrm{T}}$ are plotted. It can be seen that for all variations of the two parameters concentration and temperature $D_{T}$ follow roughly the same trend. Between the monomer and the dimer the sign of the coefficient changes. The values for the two different concentrations are equal within error. For the measurement series at $30^{\circ} \mathrm{C}$ and $40^{\circ} \mathrm{C}$ the value of $D_{T}$ increases slightly between the dimer and the trimer. Already the value of $D_{T}$ for the dimer is close to the value of the polymer PEO in ethanol with little addition of water ${ }^{26}$, indicated by the dashed line in the plot. Simulations by Zhang et al. ${ }^{24}$ show that the plateau in the thermal diffusion coefficient is usually reached at chain lengths of around two to three times the persistence length.The persistence length, $l_{p}$, of PEO in water can be calculated from the characteristic ratio, $C_{\infty}=5.2$ determined by Kawaguchi et al. ${ }^{30}$ using the well known expression $l_{p}=(b / 2)\left(C_{\infty}+1\right)$ given in textbooks ${ }^{31}$.
Assuming a bond length of $b=1.54 \AA$ we find $l_{p}=4.8 \AA$, which corresponds to $4 / 3 \approx 1.33$ monomers, if we assume that a monomer can be characterized by the oxide to oxide length of roughly $3.6 \AA$. That this is a resonable assumptions has also been experimentally confirmed ${ }^{32}$. A comparison with data of glycols measured in aqueous solution by Chan $e t$ al. is shown in Fig. 4. It can be seen that the plateau of $D_{T}$ for the aqueous solution is approached between three and four monomers, which corresponds to a approximate length of $10.8-11.4 \AA$, whereas two to three times $l_{p}$ is equivalent to $9.6-11.4 \AA$. Thus, the trend of the simulation data is met by the experimental data. For glycols in ethanol the plateau is already reached for the dimer. This result is reasonable because in ethanol there will be no hydration layer formed, hence, possibly reducing the persistence length to one monomer. Due to the insolubility of the higher molecular weight polyethylenglycols in ethanol no experimental data are available. The difference between the behavior in water and in ethanol might be explained by the following. In water the chain length behavior is governed by the strength of the hydrogen bonds. With increasing chain length of the glycol at constant molar fraction the hydrogen bonding gets weaker, hence leading to an increasing Soret coefficient, similar to the temperature behavior reported for PNiPAm by Kita et al. ${ }^{33}$. In ethanol the behavior might be governed mainly by the quality of the solvent. The longer the chains of glycol get, the worse is ethanol as a solvent, resulting finally in teh polymer which is not soluble at all in pure ethanol. Hence, the Soret coefficient decreases with increasing chain length.

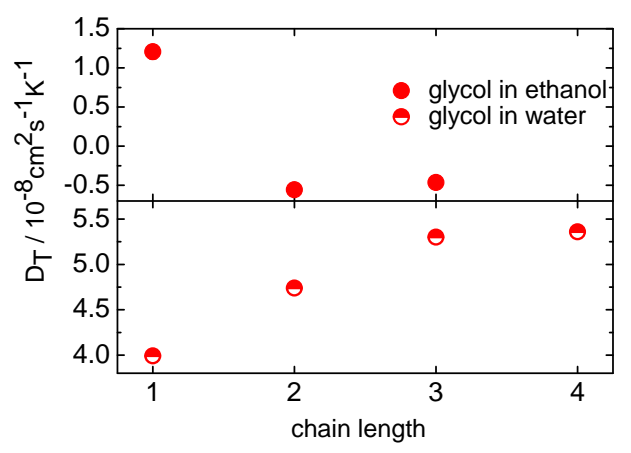

Fig. 4 Thermal diffusion coefficient $D_{T}$ as function of chain length. The upper part shows $D_{\mathrm{T}}$ of glycols in ethanol measured in this work, the lower part data from Chan et al. ${ }^{34}$ for different glycols in water. Both measurements series were carried out at $20 \%$ molar solution at $40{ }^{\circ} \mathrm{C}$.

The mass diffusion coefficient $D$, as shown in the middle of Fig. 3, shows in all cases, except the $10 \%$ molar solution at $30{ }^{\circ} \mathrm{C}$, a linear behavior. For the Soret coefficient $S_{\mathrm{T}}$, seen in 
the upper part Fig. 3 the situation is similar to the behavior of $D_{\mathrm{T}}$. The errors are comparably small and the values for the two concentrations are again equal within error. A closer look at the temperature dependence yields that the value of $S_{\mathrm{T}}$ is slightly decreasing with higher temperature.

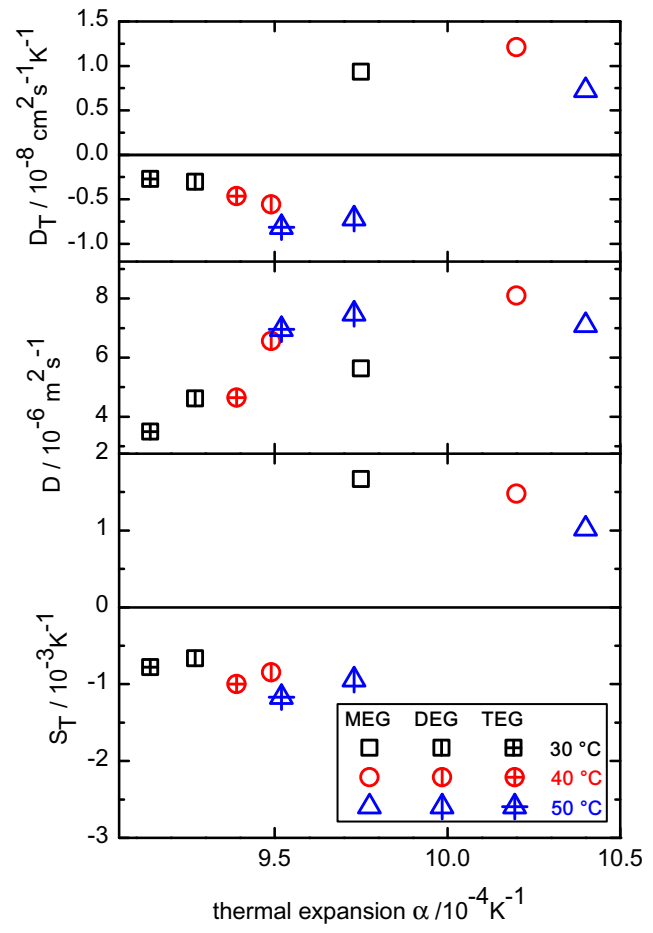

Fig. 5 Soret coefficient $S_{\mathrm{T}}$, mass diffusion coefficient $D$ and thermal diffusion coefficient $D_{\mathrm{T}}$ as function of the thermal expansion $\alpha$ for the $20 \%$ solution. For all three temperatures $S_{\mathrm{T}}$ increases almost linearly with $\alpha, D$ and $D_{\mathrm{T}}$ also increase with $\alpha$. The symbols with no, one and two bars correspond to mon- di and tri-mer, respectively.

In Fig. 5 the diffusion coefficient $D_{\mathrm{T}}$, the mass diffusion coefficient $D$ and the Soret coefficient $S_{\mathrm{T}}$ are plotted over the thermal expansion $\alpha . D$ and $S_{\mathrm{T}}$ clearly increase with increasing $\alpha$, although the data is not as linear as for previous data on saccharides for example ${ }^{20}$. It may be possible that the nonlinearity can be attributed the sign change of $D_{\mathrm{T}}$ and $S_{\mathrm{T}}$ between the monomer and the dimer, which is reported for the before mentioned data.

Also the plot of the thermal diffusion coefficient $D_{\mathrm{T}}$ over the ratio of thermal expansion, $\alpha$, and kinematic viscosity $v$, as shown in Fig. 6 gives no better linearity. This results are in contrast to the results for alkanes ${ }^{15}$ and sugars ${ }^{19,20}$. For the non-polar as well as for the polar aqueous system an excellent linear dependence of $D_{\mathrm{T}}$ with $\alpha / v$ was found. We can only speculate, why this empirical correlation, which is also sup-

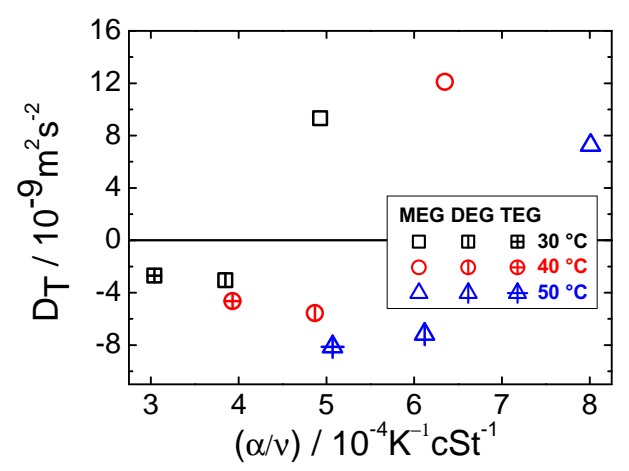

Fig. 6 Thermal diffusion $D_{\mathrm{T}}$ plotted over the ratio of thermal expansion $\alpha$ over the kinematic viscosity $v$ for the $20 \%$ molar solution. Although the increase in thermal diffusion seems not to be linear, in general it increases with increasing ratio of $\alpha$ and $v$. The symbols with no, one and two bars correspond to mon- di and tri-mer, respectively.

ported by theoretical arguments ${ }^{35}$, fails for this aqueous system. The most striking difference compared to the previous investigated systems, is the occurrence of a sign change between the glycol and diethylenglycol. Often sign changes are related to a structural change in the solutions such as a break down of a hydrogen bond network ${ }^{26,36}$. This will certainly also change the dependence on the thermal expansion coefficient. The measurements for the diethylen- and triethylenglycol indicate that for higher glycols a linear dependence might be expected, but due to the limited solubility of the higher glycols in ethanol experiments become difficult and for very higher molar masses impossible.

\section{Conclusions}

In this paper we presented the thermal diffusion coefficients of monoethylenglycol, diethylenglycol and triethylenglycole in pure ethanol. We found that the Soret coefficients and the thermal diffusion coefficients strongly depend on the chain length of the glycol. The $D_{\mathrm{T}}$ values coincide already between the dimer and the trimer with the value of the corresponding polymer PEG. Due to the short persistence length of the system of the order of one monomer the plateau value of $D_{\mathrm{T}}$ is already reached for the dimer, which is in accordance with the prediction of molecular dynamic simulation results ${ }^{24}$. We were not able to confirm the empirical correlation between the thermal diffusion coefficient and the ratio of the thermal expansion coefficient and the kinematic viscosity. We suspect that the sign change of the Soret coefficient between the monomer and the dimer at constant molar fraction might be responsible 
for this behavior. Interestingly, neither the temperature nor the concentration of the prepared solutions seems to have a large impact on the measured coefficients, at least in the regime where the measurements were conducted. This is extremely appealing for longtime experiments or experiments for which the samples have to prepared long before the measurements, as slight or even rather big deviations of the concentration of a given solution, due to evaporation of one component yields only small changes in the measured result.

\section{Acknowledgments}

We would like to thank Pablo Blanco and Bastian Arlt for many fruitful discussions and Hartmut Kriegs for technical support. Financial support due to the Deutschge Forschungsgemeinschaft grant Wi 1684 is gratefully acknowledged.

\section{References}

1 D. Braun and A. Libchaber, Phys. Rev. Lett., 2002, 89, $2-5$.

2 A. Bahat, I. Tur-Kaspa, A. Gakamsky, L. Giojalas, C. H. Breitbart and M. Eisenbach, Nat. Med., 2003, 9, 1-2.

3 C. Angeli and E. Leonardi, Int. J. Heat Mass Transfer, 2005, 48, 46334639.

4 F. Montel, G. Cailett, A. Pucheu and J. Caltagirone, Mar. Pet. Geol., 1993, 10, 51-57.

5 C. Debuschewitz and W. Köhler, Phys. Rev. Lett., 2001, 87, 3-6.

6 B.-J. de Gans, R. Kita, S. Wiegand and J. Luettmer Strathmann, Phys. Rev. Lett., 2003, 91, 245501.

7 S. N. Semenov and M. E. Schimpf, Phys. Rev. E, 2005, 72, Art. No. 041202.

8 G. Galliero, M. Bugel, B. Duguay and F. Montel, J. Non-Equilib. Thermodyn., 2007, 32, 251-258.

9 P. A. Artola, B. Rousseau and G. Galliero, J. Am. Chem. Soc., 2008, 130, 10963-10969.

10 M. Hartung, J. Rauch and W. Khler, J. Chem. Phys., 2006, 125, 214904.

11 P. Polyakov, J. Luettmer-Strathmann and S. Wiegand, J. Phys. Chem. B, 2006, 110, 26215-26224.

12 G. Wittko and W. Köhler, Europhys. Lett., 2007, 78, 46007.

13 P. Blanco, M. Bou-Ali, J. Platten, J. Madariaga, P. Urteaga and C. Santamaría, J. Non-Equilib. Thermodyn., 2007, 32, 309-317.

14 P. Blanco, P. Polyakov, M. M. Bou-Ali and S. Wiegand, J. Phys. Chem. $B, 2008,112,8340-8345$.

15 P. Blanco, M. Mounir Bou-Ali, J. K. Platten, P. Urteaga, J. a. Madariaga and C. Santamaria, J. Chem. Phys., 2008, 129, 174504.

16 J. Rauch and W. Köhler, Macromolecules, 2005, 38, 3571-3573.

17 H. Ning, J. Buitenhuis, J. K. G. Dhont and S. Wiegand, J. Chem. Phys., 2006, 125, 204911.

18 M. Braibanti, D. Vigolo and R. Piazza, Phys. Rev. Lett., 2008, 100, year.

19 P. Blanco and S. Wiegand, J. Phys. Chem. B, 2010, 114, 2807-2813.

20 P. Blanco, H. Kriegs, B. Arlt and S. Wiegand, J. Phys. Chem. B, 2010, 114, 10740-10747.

21 F. Brochard and P. de Gennes, C.R. Acd. Sci. Paris, Serie II, 1981, 293, 1025.

22 A. Würger, Phys. Rev. Lett., 2009, 102, 078302.

23 D. Stadelmaier and W. Köhler, Macromolecules, 2008, 41, 6205-6209.

24 M. Zhang and F. Müller-Plathe, J. Chem. Phys., 2006, 125, 124903.
25 J. Chan, J. Popov, S. Kolisnek-Kehl and D. Leaist, J. Solution Chem., 2003, 32, 197-214.

26 R. Kita, S. Wiegand and J. Luettmer Strathmann, J. Chem. Phys., 2004, 121, 3874-3885.

27 K. Thyagarajan and P. Lallemand, Opt. Commun., 1978, 26, 54-57.

28 W. Köhler and B. Müller, J. Chem. Phys., 1995, 103, 4367-4370.

29 W. Köhler, J. Chem. Phys., 1993, 98, 660-668.

30 S. Kawaguchi, G. Imai, J. Suzuki, a. Miyahara, T. Kitano and K. Ito, Polymer, 1997, 38, 2885-2891.

31 H.-G. Elias, Macromolecules: Volume 3: Physical Structures and Properties, Wiley-VCH, Weinheim, 2008, vol. 3.

32 K. Kenworthy, K. Hristova, D. Needham and T. J. McIntosh, Biophys. J., 1995, 68, 1921-36.

33 R. Kita, P. Polyakov and S. Wiegand, Macromolecules, 2007, 40, 1638 1642 .

34 J. Chan, J. Popov, S. Kolisnek-Kehl and D. Leaist, J. Solution Chem., 2003, 32, 197-214.

35 H. Brenner, Phys. Rev. E, 2006, 74, 036306.

36 H. Ning and S. Wiegand, J. Chem. Phys., 2006, 125, 221102. 